Pacific

Journal of

Mathematics

CONSTRUCTING FAMILIES OF LONG CONTINUED FRACTIONS

DANIEL J. MADDEN

Volume $198 \quad$ No. 1

March 2001 


\title{
CONSTRUCTING FAMILIES OF LONG CONTINUED FRACTIONS
}

\author{
DANIEL J. MADDEN
}

\begin{abstract}
This paper describes a method of constructing an unlimited number of infinite families of continued fraction expansions of the square root of $D$, an integer. The periods of these continued fractions all have identifiable sub patterns repeated a number of times according to certain parameters. For example, it is possible to construct an explicit family for the square root of $D(k, l)$ where the period of the continued fraction has length $2 k l-2$. The method is recursive and additional parameters controlling the length can be added.
\end{abstract}

\section{Section 1.}

In the last 20 years (starting with an example by D. Shanks), more and more examples of families of quadratic surds with unbounded continued fraction length have appeared. A long list of explicit continued fractions discovered by L. Bernstein, C. Levesque and G. Rind is given in $[\mathbf{L R}]$. H.C. Williams $[\mathbf{W}]$, T. Azuhatu $[\mathbf{A}]$ and many others have added to this list over the years. In $[\mathbf{v d P}]$, A. van der Poorten gives several examples as he illustrates how matrices not only make such expansions more manageable, but also are an integral part of the theory of continued fractions. In this note, we describe a method of constructing an unlimited number of distinct families of expansions using two matrix identities.

Typically in the known examples, a family of surds is given in terms of several parameters of which one is explicitly connected with the length of the period in the partial quotients. For instance, an integer $d(k, n), \sqrt{d(k, n)}$ might have the form

$$
\left[n, \overline{a_{1}, b_{1}, c_{1}, d_{1}, a_{2}, b_{2}, c_{2}, d_{2}, a_{3}, b_{2}, c_{2}, d_{2}, \ldots, a_{k}, b_{k}, c_{k}, d_{k}, 2 n}\right]
$$

where the partial quotients $a_{i}, b_{i}, c_{i}, d_{i}$ are given as formula in terms of $n$ and $i$. The internal structure of the repeating quotients contains a recurring pattern of length 4 repeated $k$ times.

In this paper we will describe methods for constructing families of integer square roots where two or more parameters control different aspects of the period length of the continued fraction expansion; i.e., $\sqrt{d\left(k_{1}, k_{2}, n\right)}$ produces a period containing a recurring pattern of length $k_{2}$ repeated $k_{1}$ 
times. Further we will see how to produce surds with periods of ever increasing complexity. We will describe a method that takes the period of one example and inserts it inside a more complicated recurring pattern. For example, starting with a sequence $\vec{a}$ of length $k_{2}$ taken from one continued fraction, we can construct another continued fraction that involves a recurring pattern $b_{i}, \vec{a}, c_{i}$ that appears $k_{1}$ times in the repeating part. The process is recursive, and it can be used to produce ever more elaborate sequences of partial quotients. Finally, our method will allow us to choose the partial quotients to be of any size so our fractions can be chosen to limit the occurrence of small partial quotients. While there is no end to the number and complexity of families our methods can produce, they become increasingly difficult to write down explicitly in terms of the controlling parameters.

The notation that follows gets rather involved, and we will be forced to set some conventions just to make things readable. To start we will no longer overline the repeating quotients in the expansion a quadratic surd, and agree that

$$
\sqrt{d}=\left[a_{0} ; a_{1}, a_{2}, \ldots \ldots \ldots, a_{n}\right]
$$

means that the quotients $a_{1}, a_{2}, \ldots \ldots \ldots, a_{n}$ repeat. We will still use

$$
\left[a_{0} ; a_{1}, a_{2}, \ldots \ldots \ldots, a_{n}\right]
$$

for the finite continued fraction in hopes that the context will allow the correct interpretation.

Suppose we have a matrix

$$
N=\left(\begin{array}{cc}
0 & 1 \\
1 & a_{1}
\end{array}\right)\left(\begin{array}{cc}
0 & 1 \\
1 & a_{2}
\end{array}\right) \cdots\left(\begin{array}{cc}
0 & 1 \\
1 & a_{n}
\end{array}\right) .
$$

We will use the shorthand notation $N=\left\{a_{1}, a_{2}, \ldots \ldots, a_{n}\right\}$ to express such a product. We will write $\vec{N}$ to denote the sequence $a_{1}, a_{2}, \ldots \ldots, a_{n}$. We will denote the reverse sequence as $\overleftarrow{N}$ which could just as well be written as $\overrightarrow{N^{T}}$.

Any such matrix can be viewed as a fractional linear functions, and we will denote this action as

$$
\left(\begin{array}{ll}
s & t \\
u & v
\end{array}\right)[x]=\frac{s x+t}{u x+v} .
$$

Of course, the composition of functions corresponds to matrix multiplication.

To set out the method used in the proofs, we will begin with an example. This example will illustrate the steps used in this paper to evaluate a continued fraction, and allow readers to reconcile the layout of these steps with their own style. The example we will use is in one of the sequences we will construct later, $\sqrt{31}$. In this case, the expansion claimed is

$$
\sqrt{31}=[5 ; \overline{1,1,3,5,3,1,1,10}] .
$$


We can compute the convergents of the first cycle using the familiar PQ chart:

\begin{tabular}{|l|l|l|l|l|r|r|r|r|r|r|r|}
\hline & & & 5 & 1 & 1 & 3 & 5 & 3 & 1 & 1 & 10 \\
\hline $\mathrm{Q}$ & 1 & 0 & 1 & 1 & 2 & 7 & 37 & 118 & 155 & 273 & 2885 \\
\hline $\mathrm{P}$ & 0 & 1 & 5 & 6 & 11 & 39 & 206 & 657 & 863 & 1520 & 16063 \\
\hline
\end{tabular}

For our purposes it is better to change the last quotient in this chart from 10 to 5 , then the last two columns look like

\begin{tabular}{|r|r|}
\hline 1 & 5 \\
\hline 273 & 1520 \\
\hline 1520 & 8463 \\
\hline
\end{tabular}

This calculation can be expressed in our matrix notation as

$$
M=\{5 ; 1,1,3,5,3,1,1,5\}=\left(\begin{array}{cc}
273 & 1520 \\
1520 & 8463
\end{array}\right) .
$$

Because the continued fraction repeats, the value of this fraction satisfies the quadratic equation

$$
M[x]=\frac{273 x+1520}{1520 x+8463}=\frac{1}{x} .
$$

This reduce to

$$
273 x^{2}=8463
$$

or

$$
x^{2}=31 .
$$

Further we can obtain the smallest solutions to Pell's equation from these last two columns

$$
1520^{2}-273^{2} \cdot 31=1 .
$$

In general, if $\sqrt{d}=\left[a_{0} ; a_{1}, a_{2}, \ldots \ldots \ldots, 2 a_{0}\right]$, then the corresponding matrix $M=\left\{a_{0}, a_{1}, a_{2}, \ldots \ldots \ldots, a_{0}\right\}$ must have the form

$$
M=\left(\begin{array}{cc}
s & t \\
t & s d
\end{array}\right)
$$

where

$$
s^{2} d-t^{2}= \pm 1 \text {. }
$$

Then $\sqrt{d}$ is the solution to the quadratic equation:

$$
M[x]=\frac{1}{x} .
$$


Further,

$$
\left(\begin{array}{cc}
s & t \\
t & s d
\end{array}\right)\left(\begin{array}{cc}
\sqrt{d} & -\sqrt{d} \\
1 & 1
\end{array}\right)=\left(\begin{array}{cc}
1 & 1 \\
\sqrt{d} & -\sqrt{d}
\end{array}\right)\left(\begin{array}{cc}
t+s \sqrt{d} & 0 \\
0 & t-s \sqrt{d}
\end{array}\right) .
$$

If $d \not \equiv 1(\bmod 4)$ and is square free, $t+s \sqrt{d}$ is the fundamental unit of $\mathbb{Q}(\sqrt{d})$.

\section{Section 2.}

All of our examples hinge on the reduction of a matrix product of the form

$$
M=A\left(N_{k-1} N_{k-2} N_{k-3} \cdots N_{2} N_{1} N_{0}\right) A\left(N_{k-1} N_{k-2} N_{k-3} \cdots N_{2} N_{1} N_{0}\right)^{T} A .
$$

Suppose we have a family of matrices of the form

$$
N_{t}=\left(\begin{array}{cc}
p & 2 b r^{t} \\
b r^{k-1-t} & q
\end{array}\right)
$$

where

$$
p q-2 b^{2} r^{k-1}=\epsilon= \pm 1
$$

and

$$
q r-p=2 b m \quad \text { for some } m \text {. }
$$

The product $N_{k-1} N_{k-2} N_{k-3} \cdots N_{2} N_{1} N_{0}$ can be reduced quickly. If

$$
\left(\begin{array}{ll}
1 & 0 \\
0 & r
\end{array}\right)
$$

it is easy to see that $N_{t}=C^{-t} N_{0} C^{t}$. So our first reduction is

$$
N_{k-1} \cdots N_{1} N_{0}=C^{-k}\left(C N_{0}\right)^{k} \text {. }
$$

Now consider

$$
K=C N_{0}=\left(\begin{array}{cc}
p & 2 b \\
b r^{k} & q r
\end{array}\right) .
$$

The fixed value of $K^{T}[x], \alpha$, satisfies

$$
2 b \alpha^{2}+(q r-p) \alpha-b r^{k}=0 .
$$

So if

$$
d=m^{2}+2 r^{k}
$$

and

$$
\alpha=\frac{-m+\sqrt{d}}{2},
$$

then $\alpha$ and its conjugate $\bar{\alpha}$ are fixed values of $K^{T}[x]$. 
Next, let

$$
E=\left(\begin{array}{cc}
\sqrt{d} & -\sqrt{d} \\
1 & 1
\end{array}\right)
$$

and

$$
A=\left(\begin{array}{cc}
0 & 1 \\
1 & m
\end{array}\right) .
$$

Now one simply verifies that

$$
C^{-k} A E=\left(\begin{array}{cc}
1 & 1 \\
\alpha^{-1} & \bar{\alpha}^{-1}
\end{array}\right)=\left(\begin{array}{cc}
\alpha & \bar{\alpha} \\
1 & 1
\end{array}\right)\left(\begin{array}{cc}
\alpha^{-1} & 0 \\
0 & \bar{\alpha}^{-1}
\end{array}\right) .
$$

Next

$$
K^{T}\left(\begin{array}{cc}
\alpha & \bar{\alpha} \\
1 & 1
\end{array}\right)=\left(\begin{array}{cc}
2 b \alpha^{2}+q r \alpha & 2 b \bar{\alpha}^{2}+q r \bar{a} l \\
2 b \alpha+q r & 2 b \bar{\alpha}+q r
\end{array}\right) .
$$

So we have

$$
K^{T}\left(\begin{array}{cc}
\alpha & \bar{\alpha} \\
1 & 1
\end{array}\right)=\left(\begin{array}{cc}
\alpha & \bar{\alpha} \\
1 & 1
\end{array}\right)\left(\begin{array}{ll}
\beta & 0 \\
0 & \bar{\beta}
\end{array}\right)
$$

where

$$
\beta=2 b \alpha+q r=2 b \alpha+p=p+2 b(m+\alpha)=p-2 b \bar{\alpha} .
$$

Now $\beta$ and its conjugate $\bar{\beta}$ are the eigenvalues of $K^{T}$ (and consequently $K)$. Immediately,

$$
\left(K^{T}\right)^{k}=\left(\begin{array}{cc}
\alpha & \bar{\alpha} \\
1 & 1
\end{array}\right)=\left(\begin{array}{cc}
\alpha & \bar{\alpha} \\
1 & 1
\end{array}\right)\left(\begin{array}{cc}
\beta^{k} & 0 \\
0 & \bar{\beta}^{k}
\end{array}\right) .
$$

Next

$$
A\left(\begin{array}{cc}
\alpha & \bar{\alpha} \\
1 & 1
\end{array}\right)=\left(\begin{array}{cc}
1 & 1 \\
m+\alpha & m+\bar{\alpha}
\end{array}\right)=\left(\begin{array}{cc}
1 & 1 \\
-\bar{\alpha} & -\alpha
\end{array}\right) .
$$

This in turn diagonalizes $K$ :

$$
K\left(\begin{array}{cc}
1 & 1 \\
-\bar{\alpha} & -\alpha
\end{array}\right)=\left(\begin{array}{ll}
\beta & 0 \\
0 & \bar{\beta}
\end{array}\right) .
$$

Again

$$
K^{k}\left(\begin{array}{cc}
1 & 1 \\
-\bar{\alpha} & -\alpha
\end{array}\right)=\left(\begin{array}{cc}
1 & 1 \\
-\bar{\alpha} & -\alpha
\end{array}\right)\left(\begin{array}{cc}
\beta^{k} & 0 \\
0 & \bar{\beta}^{k}
\end{array}\right) .
$$

Finally

$$
A C^{-k}\left(\begin{array}{cc}
1 & 1 \\
-\bar{\alpha} & -\alpha
\end{array}\right)=\left(\begin{array}{cc}
1 & 1 \\
\sqrt{d} & -\sqrt{d}
\end{array}\right)\left(\begin{array}{cc}
\frac{1}{2 \alpha} & 0 \\
0 & \frac{1}{2 \alpha}
\end{array}\right) .
$$


Now the whole point of this is

$$
N_{k-1} N_{k-2} N_{k-3} \cdots N_{2} N_{1} N_{0}=C^{-k}\left(C N_{0}\right)^{k}=C^{-k} K^{k} .
$$

So if we define $M$ as

$$
M=A\left(N_{k-1} N_{k-2} N_{k-3} \cdots N_{2} N_{1} N_{0}\right) A\left(N_{k-1} N_{k-2} N_{k-3} \cdots N_{2} N_{1} N_{0}\right)^{T} A
$$

then

$$
\begin{aligned}
M E & =A C^{-k} K^{k} A\left(C^{-k} K^{k}\right)^{T} A E \\
& =A C^{-k} K^{k} A\left(K^{T}\right)^{k} C^{-k} A E \\
& =\left(\begin{array}{cc}
1 & 1 \\
\sqrt{d} & -\sqrt{d}
\end{array}\right)\left(\begin{array}{cc}
\beta^{2 k} & 0 \\
0 & \bar{\beta}^{2 k}
\end{array}\right)\left(\begin{array}{cc}
\frac{1}{2 \alpha^{2}} & 0 \\
0 & \frac{1}{2 \alpha^{2}}
\end{array}\right) .
\end{aligned}
$$

This implies

$$
M\left(\begin{array}{cc}
\sqrt{d} & -\sqrt{d} \\
1 & 1
\end{array}\right)=\left(\begin{array}{cc}
1 & 1 \\
\sqrt{d} & -\sqrt{d}
\end{array}\right)\left(\begin{array}{ll}
\gamma & 0 \\
0 & \bar{\gamma}
\end{array}\right),
$$

and so our identity is immediately useful in establishing the continued fraction expansion of $\sqrt{d}$.

Proposition 1. Suppose we have a family of matrices of the form

$$
N_{i}(q, b, m, r)=\left(\begin{array}{cc}
q r-2 b m & 2 b r^{i} \\
b r^{k-1-i} & q
\end{array}\right) \quad i=0,1,2, \ldots, k-1 .
$$

Further suppose that each $N_{i}=\{a(i, 1), a(i, 2), \ldots, a(i, n)\}$ where $a(i, j)$ are integers. If $d(m, r)=m^{2}+2 r^{k}$, then the following is a valid continued fraction expansion of $\sqrt{d(m, r)}$ :

$$
\sqrt{d(m, r)}=\left[m ; \overrightarrow{N_{0}}, \overrightarrow{N_{1}}, \ldots, \overleftarrow{N_{k-1}}, \ldots, \overleftarrow{N_{1}}, \overleftarrow{N_{0}}, 2 m\right]
$$

Further if $d(m, r)$ is square free and $d \equiv 2$ or $3(\bmod 4)$, then the fundamental unit of $\mathbb{Q}(\sqrt{d(m, r)})$ is

$$
\frac{\beta^{2 k}}{2 \alpha^{2}}
$$

where

$$
\alpha=\frac{-m+\sqrt{d(m, r)}}{2} \text { and } \beta=q r-2 b m-2 b \bar{\alpha}
$$




\section{Section 3.}

The calculation above can be used directly to produce examples of continued fractions of quadratic surds with arbitrarily long repeating pattern. Let $b, n$ and $k$ be any natural numbers. We have

$$
\begin{aligned}
& \sqrt{\left(b(2 b n+1)^{k}+n\right)^{2}+2(2 b n+1)^{k}} \\
&=\left[b(2 b n+1)^{k}+n ;\right. \\
& b, 2 b(2 b n+1)^{k-1}, b(2 b n+1), 2 b(2 b n+1)^{k-2}, b(2 b n+1)^{2}, \ldots, \\
& b(2 b n+1)^{k-2}, 2 b(2 b n+1)^{1}, b(2 b n+1)^{k-1}, 2 b, \\
& b(2 b n+1)^{k}+n, \\
& 2 b, b(2 b n+1)^{k-1}, 2 b(2 b n+1), b(2 b n+1)^{k-2}, 2 b(2 b n+1)^{2}, \ldots, \\
&\left.2 b(2 b n+1)^{k-2}, b(2 b n+1)^{1}, 2 b(2 b n+1)^{k-1}, b, 2 b(2 b n+1)^{k}+2 n\right] .
\end{aligned}
$$

The length of the repeating pattern is $4 k+2$. This expansion comes from the matrix product

$$
\left(\begin{array}{cc}
0 & 1 \\
1 & b r^{k-1-t}
\end{array}\right)\left(\begin{array}{cc}
0 & 1 \\
1 & 2 b r^{t}
\end{array}\right)=\left(\begin{array}{cc}
1 & 2 b r^{t} \\
b r^{k-1-t} & 2 b^{2} r^{k-1}+1
\end{array}\right)
$$

which is in the form used in Section 2 provided $q r-p=2 b^{2} r^{k}+r-1$ is divisible by $2 b$. If we choose $r=2 b n+1$, this condition will be met. In the proposition, we wrote $q r-p=2 b m$. Keeping this notation

$$
m=b r^{k}+n=b(2 b n+1)^{k}+n .
$$

This leads us to the surd

$$
\sqrt{d}=\sqrt{m^{2}+2 r^{k}}=\sqrt{\left(b(2 b n+1)^{k}+n\right)^{2}+2(2 b n+1)^{k}} .
$$

Note that every partial quotient in this family is greater than or equal to $b$, so this gives infinite families of quadratic surds with all partial quotients arbitrarily large.

In this example, $r=2 b n+1$ is odd, so $d=m^{2}+2 r^{k} \equiv 2$ or $1(\bmod 4)$. If

$$
d=\left(b(2 b n+1)^{k}+n\right)^{2}+2(2 b n+1)^{k}
$$

is square free, the fundamental unit of $\mathbb{Q}(\sqrt{d})$ comes directly from the continued fraction. This unit is

where

$$
\frac{\beta^{2 k}}{2 \alpha^{2}}
$$

$$
\alpha=\frac{1}{2}\left(-b(2 b n+1)^{k}-n+\sqrt{d}\right)
$$


and

$$
\beta=1-2 b \bar{\alpha}=1+b n+b^{2}(2 b n+1)^{k}+b \sqrt{d} .
$$

The next example is similar to examples found in $[\mathbf{v d P}]$. Here $\sqrt{d(k, b, n)}$ has length $6 k+2$. Let $b, n$ and $k$ be any natural numbers,

$$
\begin{aligned}
& \sqrt{\left(b(2 b n-1)^{k}-n\right)^{2}+2(2 b n-1)^{k}} \\
& =\left[b(2 b n-1)^{k}-n ; b-1, \quad 1, \quad 2 b(2 b n-1)^{k-1}-1,\right. \\
& b(2 b n-1)-1, \quad 1, \quad 2 b(2 b n-1)^{k-2}-1, \\
& b(2 b n-1)^{2}-1, \quad 1, \quad 2 b(2 b n-1)^{k-3}-1, \\
& b(2 b n-1)^{k-2}-1, \quad 1, \quad 2 b(2 b n-1)-1, \\
& b(2 b n-1)^{k-1}-1, \quad 1, \quad 2 b-1, \\
& b(2 b n-1)^{k}-n, \\
& 2 b-1, \quad 1, \quad b(2 b n-1)^{k-1}-1 \\
& 2 b(2 b n-1)^{1}-1, \quad 1, \quad b(2 b n-1)^{k-2}-1, \\
& 2 b(2 b n-1)^{k-2}-1,1, \quad b(2 b n-1)-1, \\
& 2 b(2 b n-1)^{k-1}-1,1, \quad b-1, \\
& \left.2 b(2 b n-1)^{k}-2 n\right] \text {. }
\end{aligned}
$$

This identity is verified by computing the matrix product

$$
\left(\begin{array}{cc}
0 & 1 \\
1 & b r^{k-1-t}-1
\end{array}\right)\left(\begin{array}{ll}
0 & 1 \\
1 & 1
\end{array}\right)\left(\begin{array}{cc}
0 & 1 \\
1 & 2 b r^{t}-1
\end{array}\right)=\left(\begin{array}{cc}
1 & 2 b r^{t} \\
b r^{k-1-t} & 2 b^{2} r^{k-1}-1
\end{array}\right) \text {. }
$$

For $r=2 b n-1$, this gives $q r-p=2 b^{2} r^{k}-r-1=2 b\left(b r^{r}-n\right)$.

Once again $r$ is odd, and we can get information about units in quadratic fields directly from the proof of this identity. If $d$ in this example is squarefree, the fundamental unit of $\mathbb{Q}[\sqrt{d}]$ is

$$
\frac{\beta^{2 k}}{2 \alpha^{2}}
$$

where $\alpha=\frac{1}{2}\left(-b(2 b n-1)^{k}+n+\sqrt{d}\right)$, and $\beta=1-2 b \bar{\alpha}$.

\section{Section 4 .}

In the second example above, we found a family of expansions in which the repeating partial quotients contain a recurring pattern of the form 
$A_{i}, 1, B_{k-i}$. Next we provide a method for producing expansions where the repeating pattern contains recurring patterns of the form $A_{i}, a_{1}, a_{2}, \ldots, a_{n}$, $B_{k-i}$. Unfortunately, the $a_{i}$ cannot be chosen arbitrarily. We will see later exactly how to choose proper $a_{i}$.

Suppose you have an integral matrix product of the form

$$
\left\{a_{1}, a_{2}, \ldots \ldots \ldots, a_{n}\right\}=\left(\begin{array}{cc}
u & v \\
2 v-\delta w & w
\end{array}\right),
$$

where $\delta=0$ or 1 . The determinant of this matrix is

$$
\epsilon=(-1)^{n}=u w-2 v^{2}+\delta v w .
$$

If we choose the following values for $b, r, m, A_{i}$, and $B_{i}$, then we can produce a family of matrices like those in Proposition 1 from

$$
N_{i}=\left\{v A_{k-1-i}, a_{1}, a_{2}, \ldots \ldots \ldots, a_{n}, B_{i}\right\} .
$$

For any value of $l \geq 0$, let

$$
\begin{aligned}
b & =b(u, v, w, \delta ; l)=v \\
r & =r(u, v, w, \delta ; l)=\epsilon\left(w^{2}-2 v^{2}\right)+2 l w v \\
A_{i} & =A_{i}(u, v, w, \delta ; l)=\frac{r^{i}-1}{w} \\
B_{i} & =B_{i}(u, v, w, \delta ; l)=2 v A_{i}+\delta
\end{aligned}
$$

and

$$
m=m(u, v, w, \delta ; l)=v A_{k}+\epsilon l .
$$

Now it may not be immediately clear that the $A_{i}$ are all integers, but they are since

$$
\begin{aligned}
r-1 & =\epsilon\left(w^{2}-2 v^{2}\right)+2 l w v-1 \\
& =\epsilon\left(w^{2}+\epsilon-u w-\delta v w\right)+2 l w v-1 \\
& =\epsilon\left(w^{2}-u w-\delta v w\right)+2 l w v .
\end{aligned}
$$

Thus

$$
\frac{r-1}{w}=\epsilon(w-u-\delta v)+2 l v .
$$

These values are set so that we have the required form:

$$
\left(\begin{array}{cc}
0 & 1 \\
1 & v A_{k-1-i}
\end{array}\right)\left(\begin{array}{cc}
u & v \\
2 v-\delta w & w
\end{array}\right)\left(\begin{array}{cc}
0 & 1 \\
1 & B_{i}
\end{array}\right)=\left(\begin{array}{cc}
w & 2 b r^{i} \\
b r^{k-1-i} & q
\end{array}\right)
$$

where

$$
q=u+(2 v-\delta w) v A_{k-1-i}+B_{i} b r^{k-1-i} .
$$


We can simplify $q$ using the fact that the determinant $q w-2 b^{2} r^{k-1}=\epsilon$. Then

$$
q=\frac{2 b^{2} r^{k-1}+\epsilon}{w} .
$$

Thus it is independent of $i$. To use Proposition 1, we need to check that $q r-w=2 b m$.

$$
\begin{aligned}
q r-w & =\frac{2 v^{2} r^{k}+\epsilon r-w^{2}}{w}=\frac{2 v^{2} r^{k}+\left(w^{2}-2 v^{2}\right)+2 \epsilon l w v-w^{2}}{w} \\
& =2 v \frac{r^{k}-1+\epsilon l v w}{w}=2 v\left(v \frac{r^{k}-1}{w}+\epsilon l\right) \\
& =2 v\left(v A_{k}+\epsilon l\right)=2 v m
\end{aligned}
$$

because $b=v$ and $m=v A_{k}+\epsilon l$.

Once we have our matrix family, we have a continued fraction expansion for

$$
\sqrt{d}=\sqrt{m^{2}+2 r^{k}}
$$

where $m=v A_{k}+\epsilon l$, and $A_{k}=\frac{r^{k}-1}{w}$. We can write

$$
d=\left(v A_{k}+\epsilon l\right)^{2}+2 w A_{k}+2=v^{2} A_{k}^{2}+2(\epsilon l v+w) A_{k}+2 .
$$

\section{Section 5 .}

Suppose we have a sequence of integers $a_{1} ; a_{2}, \ldots, a_{n}$ for which

$$
\left\{a_{1} ; a_{2}, \ldots, a_{n}\right\}=\left(\begin{array}{cc}
u & v \\
2 v-\delta w & w
\end{array}\right)
$$

where $\delta=0$ or 1 . Let $\epsilon=u w-x v=(-1)^{n}$. In the last section we saw how to use this to construct a matrix of the form in Proposition 1, and so we are led to a formal continued fraction ezpansion of a quadratic surd. With the definitions,

$$
\begin{gathered}
r=\epsilon w^{2}-2 \epsilon v^{2}+2 l w v . \\
A_{t}=\frac{r^{t}-1}{w} \\
B_{t}=2 v A_{t}+\delta \\
m=v A_{k}+\epsilon l \\
d=m^{2}+2 r^{k}=v^{2} A_{k}^{2}+2(\epsilon l v+w) A_{k}+2 \\
\alpha=\frac{-m+\sqrt{d}}{2} \\
\beta=w-2 b \bar{\alpha},
\end{gathered}
$$


we saw that

$$
N_{t}=\left\{v A_{t}, a_{1} ; a_{2}, \ldots, a_{n}, B_{k-1-t}\right\}=\left(\begin{array}{cc}
w & 2 b r^{s} \\
b r^{k-1-t} & q
\end{array}\right) .
$$

This matrix is of the type in Proposition 1, so

$$
\sqrt{d}=\left[\overrightarrow{N_{0}}, \overrightarrow{N_{1}}, \ldots, \overrightarrow{N_{k-1}}, m, \overleftarrow{N_{k-1}}, \ldots, \overleftarrow{N_{1}}, \overleftarrow{N_{0}}, 2 m\right]
$$

This almost means that, as a continued fraction,

$$
\begin{aligned}
& \sqrt{d}=\left[m ; \quad A_{0}, \quad a_{1}, a_{2}, \ldots \ldots \ldots, a_{n}, B_{k-1},\right. \\
& A_{1}, \quad a_{1}, a_{2}, \ldots \ldots \ldots, a_{n}, B_{k-2} \text {, } \\
& A_{2}, \quad a_{1}, a_{2}, \ldots \ldots \ldots, a_{n}, B_{k-3}, \\
& A_{k-2}, a_{1}, a_{2}, \ldots \ldots \ldots, a_{n}, B_{1} \\
& A_{k-1}, a_{1}, a_{2}, \ldots \ldots \ldots, a_{n}, B_{0} \\
& m \text {, } \\
& B_{0}, \quad a_{n}, a_{n-1}, \ldots \ldots \ldots, a_{1}, A_{k-1}, \\
& B_{1}, \quad a_{n}, a_{n-1}, \ldots \ldots \ldots, a_{1}, A_{k-2} \text {, } \\
& B_{2}, \quad a_{n}, a_{n-1}, \ldots \ldots \ldots, a_{1}, A_{k-3}, \\
& B_{k-2}, a_{n}, a_{n-1}, \ldots \ldots \ldots, a_{1}, A_{1} \text {, } \\
& B_{k-1}, a_{n}, a_{n-1}, \ldots \ldots \ldots, a_{1}, A_{0} \text {, } \\
& 2 m] \text {. }
\end{aligned}
$$

If $d$ is square-free and not equivalent to $1 \bmod 4$, then the calculations also lead us directly to the fundamental unit of $\mathbb{Q}[\sqrt{d}]$. It is

$$
\frac{\beta^{2 k}}{2 \alpha^{2}} \text {. }
$$

There is a problem that may arise in the above; the partial quotients in the continued fraction may not all be positive. In fact, we always have $A_{0}=0$, and when $\delta=0$, we also have $B_{0}=0$. Further, $r$ could be negative. While the identity from Proposition 1 is valid, it may not represent the correct form of the continued fraction expansion.

The offending zeros are not a major problem, since they do not change the matrix identity. They can easily be dropped using the original meaning of a continued fraction. If the sequence $[\ldots a, b, 0, d, e, \ldots]$ occurs in a valid continued fraction identity, it can be replaced by $[\ldots a, b+d, e, \ldots]$. We can use this to change our identity into the standard continued fraction of the 
surd, but we need borrow a bit from the next repeating cycle to get exactly what we want.

When $\delta=1$, only $A_{0}=0$, and we end up with an expansion of length $2(n+2) k$.

$$
\begin{aligned}
& \sqrt{d}=\left[m+a_{1} ; \quad a_{2}, \ldots \ldots \ldots, a_{n}, B_{k-1},\right. \\
& v A_{1}, \quad a_{1}, a_{2}, \ldots \ldots \ldots, a_{n}, B_{k-2}, \\
& v A_{2}, \quad a_{1}, a_{2}, \ldots \ldots \ldots, a_{n}, B_{k-3} \text {, } \\
& v A_{k-2}, a_{1}, a_{2}, \ldots \ldots \ldots, a_{n}, B_{1}, \\
& v A_{k-1}, a_{1}, a_{2}, \ldots \ldots \ldots, a_{n}, B_{0}, \\
& m \\
& B_{0} \quad a_{n}, a_{n-1}, \ldots \ldots \ldots, a_{1}, v A_{k-1} \text {, } \\
& B_{1} \quad a_{n}, a_{n-1}, \ldots \ldots \ldots, a_{1}, v A_{k-2} \text {, } \\
& B_{2} \quad a_{n}, a_{n-1}, \ldots \ldots \ldots, a_{1}, v A_{k-3}, \\
& B_{k-2}, a_{n}, a_{n-1}, \ldots \ldots \ldots, a_{1}, v A_{1} \text {, } \\
& B_{k-1}, a_{n}, a_{n-1}, \ldots \ldots \ldots, a_{2} \text {, } \\
& \left.2 m+2 a_{1}\right] \text {. }
\end{aligned}
$$

When $\delta=0$, then $A_{0}=B_{0}=0$. This adjust to an expansion with length $2(n+2) k-2$.

$$
\begin{aligned}
& \sqrt{d}=\left[m+a_{1} ; \quad a_{2}, \ldots \ldots \ldots, a_{n}, B_{k-1},\right. \\
& v A_{1}, \quad a_{1}, a_{2}, \ldots \ldots \ldots, a_{n}, B_{k-2}, \\
& v A_{2}, \quad a_{1}, a_{2}, \ldots \ldots \ldots, a_{n}, B_{k-3}, \\
& v A_{k-2}, a_{1}, a_{2}, \ldots \ldots \ldots, a_{n}, B_{1}, \\
& v A_{k-1}, a_{1}, a_{2}, \ldots \ldots \ldots, a_{n-1} \text {, } \\
& 2 a_{n}+m \text {, } \\
& a_{n-1}, \ldots \ldots \ldots, a_{1}, v A_{k-1}, \\
& B_{1}, \quad a_{n}, a_{n-1}, \ldots \ldots \ldots, a_{1}, v A_{k-2} \text {, } \\
& B_{2}, \quad a_{n}, a_{n-1}, \ldots \ldots \ldots, a_{1}, v A_{k-3} \text {, } \\
& B_{k-2}, a_{n}, a_{n-1}, \ldots \ldots \ldots, a_{1}, v A_{1} \text {, } \\
& B_{k-1}, a_{n}, a_{n-1}, \ldots \ldots \ldots, a_{2} \text {, }
\end{aligned}
$$




$$
\left.2 m+2 a_{1}\right]
$$

The multiplier $r$ is a bit more trouble because it could be negative. This can be avoided with the right choice of $l$ :

$$
r=\epsilon w^{2}-2 \epsilon v^{2}+2 l w v .
$$

Since $l$ can be any integer, $r$ can certainly be chosen positive. This quick fix will always work, but there is another method that will allow us to take $l=0$. Recall that

$$
\left(\begin{array}{cc}
u & v \\
x & w
\end{array}\right)=\left(\begin{array}{cc}
0 & 1 \\
1 & a_{1}
\end{array}\right)\left(\begin{array}{cc}
0 & 1 \\
1 & a_{2}
\end{array}\right) \cdots\left(\begin{array}{cc}
0 & 1 \\
1 & a_{n}
\end{array}\right)
$$

and that

$$
\epsilon=(-1)^{n} \text {. }
$$

If $r=\epsilon\left(w^{2}-2 v^{2}\right)$ is negative, then we can change the parity of the length of the fraction by noting that

$$
\left(\begin{array}{cc}
u & v \\
x & w
\end{array}\right)=\left\{a_{1} ; a_{2}, \ldots, a_{n-1}, a_{n}\right\}=\left\{a_{1} ; a_{2}, \ldots, a_{n-1}, a_{n}-1,1\right\} .
$$

(We use this in whichever direction is necessary.) If we arrange the $a_{1} ; a_{2}$, $\ldots, a_{n}$ so that

$$
\begin{cases}\text { if } w^{2}>2 v^{2}, & \text { then } \epsilon=1, \\ \text { if } w^{2}<2 v^{2}, & \text { then } \epsilon=-1 .\end{cases}
$$

This will guarantee that $r>0$.

All this has gotten a bit complicated; so we will summarize with our next proposition.

Proposition 2. Suppose we have a sequence of integers $a_{1}, a_{2}, \ldots, a_{n}$ for which

$$
\left\{a_{1}, a_{2}, \ldots, a_{n}\right\}=\left(\begin{array}{cc}
u & v \\
2 v-\delta w & w
\end{array}\right)=U
$$

and $\delta=0$ or 1 . From the given values of $u, v, w$ and $\delta$ and for any values of $l \geq 0, k \geq 1$, let

$$
\begin{gathered}
\epsilon=\epsilon(u, v, w, \delta)=u w-(2 v-\delta) v=(-1)^{n} \\
r=r(u, v, w, \delta ; l)=\epsilon\left(w^{2}-2 v^{2}\right)+2 l w v \\
A_{i}=A_{i}(u, v, w, \delta ; l)=\frac{r^{i}-1}{w} \\
B_{i}=B_{i}(u, v, w, \delta ; l)=2 v A_{i}+\delta \\
m=m(u, v, w, \delta ; l, k)=v A_{k}+\epsilon l
\end{gathered}
$$


and

$$
d=d(u, v, w, \delta ; l, k)=m^{2}+2 r^{k}=v^{2} A_{k}^{2}+2(\epsilon l v+w) A_{k}+2 .
$$

We can always choose $l$ large enough that $r$ is positive. However, if we arrange the $a_{1}, a_{2}, \ldots, a_{n}$ so that

$$
\begin{cases}\epsilon=1, & \text { if } w^{2}>2 v^{2}, \\ \epsilon=-1, & \text { if } w^{2}<2 v^{2},\end{cases}
$$

we can choose any $l \geq 0$.

From these choices $\sqrt{d}$ has a continued fraction expansion

$$
\begin{aligned}
\sqrt{d}=\left[m, v A_{0}, \vec{U}, B_{k-1}, v A_{1}, \vec{U}, B_{k-2}, \ldots, v A_{k-1}, \vec{U}, B_{0},\right. \\
\\
\left.m, B_{0}, \overleftarrow{U}, v A_{k-1}, \ldots, B_{k-1}, \overleftarrow{U}, v A_{0}, 2 m\right] .
\end{aligned}
$$

After zeros are removed, this is of form (1) or (2) above. The length of the repeating pattern is $2 k(n+2)+2$ minus twice the number of zeros in the set $\left\{A_{0}, B_{0}\right\}$.

If $d \equiv 2$ or $3(\bmod 4)$ and is square free, then the fundamental unit of $\mathbb{Q}(\sqrt{d})$ is

where

$$
\frac{\beta^{2 k}}{2 \alpha^{2}}
$$

$$
\alpha=\frac{-m+\sqrt{d}}{2} \text { and } \beta=w-2 v \bar{\alpha} .
$$

\section{Section 6 .}

Of course, there is now the problem of constructing a sequence of integers $a_{1}, a_{2}, \ldots, a_{n}$ for which

$$
\left(\begin{array}{cc}
0 & 1 \\
1 & a_{1}
\end{array}\right)\left(\begin{array}{cc}
0 & 1 \\
1 & a_{2}
\end{array}\right) \cdots\left(\begin{array}{cc}
0 & 1 \\
1 & a_{n}
\end{array}\right)=\left(\begin{array}{cc}
u & v \\
2 v-\delta w & w
\end{array}\right)
$$

with $\delta=0$ or 1 . (From this $u w-x v=(-1)^{n}=\epsilon$.) There are two (roughly equivalent) approaches to this.

First we can choose any rational number which in reduced form has either an even numerator or denominator.

$$
\frac{w}{v}=\left[a_{1} ; a_{2}, \ldots, a_{n}\right] .
$$

First suppose $v=2 v^{\prime}$, and let

$$
\left\{a_{1} ; a_{2}, \ldots, a_{n}\right\}=\left(\begin{array}{cc}
u & 2 v^{\prime} \\
x & w
\end{array}\right) .
$$


If we then expand the continued fraction $2 \frac{w}{v}$

$$
2 \frac{w}{v}=\left[b_{1} ; b_{2}, \ldots, b_{n}\right]
$$

we can assume

$$
\left(\begin{array}{cc}
u & v^{\prime} \\
2 x & w
\end{array}\right)=\left[b_{1} ; b_{2}, \ldots, b_{m}\right]
$$

Then

$$
\begin{aligned}
& \left\{b_{1} ; b_{2}, \ldots, b_{m}, a_{n} ; a_{n-1}, \ldots, a_{1}\right\} \\
& =\left(\begin{array}{cc}
u & v^{\prime} \\
2 x & w
\end{array}\right)\left(\begin{array}{cc}
u & 2 v^{\prime} \\
x & w
\end{array}\right)=\left(\begin{array}{cc}
u^{2}+2 v^{\prime 2} & x u+w v^{\prime} \\
2\left(x u+w v^{\prime}\right) & 2 x^{2}+w^{2}
\end{array}\right) .
\end{aligned}
$$

If $v$ is odd, and $w=2 w^{\prime}$, a similar trick works for $\frac{w}{v}$ and $\frac{w}{2 v}$.

For an example, start with $\frac{1}{2}$. This leads to the matrix product

$$
\{2,1\}=\left(\begin{array}{ll}
0 & 1 \\
1 & 2
\end{array}\right)\left(\begin{array}{ll}
0 & 1 \\
1 & 1
\end{array}\right)=\left(\begin{array}{ll}
1 & 1 \\
2 & 3
\end{array}\right)
$$

and in turn to values set in Proposition 2:

$$
\begin{aligned}
\epsilon & =1 \\
r & =7+6 l \\
A_{i} & =\frac{r^{i}-1}{3} \\
B_{i} & =2 A_{i} \\
m & =A_{k}+l
\end{aligned}
$$

and

$$
d=m^{2}+2 r^{k}=\left(\frac{(6 l+7)^{k}+3 l-1}{3}\right)^{2}+2(6 l+7)^{k}
$$

Then

gives

$$
N_{t}=\left\{\frac{(6 l+7)^{t}-1}{3}, 2,1,2 \frac{(6 l+7)^{k-1-t}-1}{3}\right\}
$$

$$
\begin{aligned}
& \sqrt{\left(\frac{(6 l+7)^{k}+3 l-1}{3}\right)^{2}+2(6 l+7)^{k}} \\
& =\left[m, \vec{N}_{0}, \vec{N}_{1}, \ldots, \vec{N}_{k-1}, m, \overleftarrow{N}_{k-1} \overleftarrow{N}_{k-2}, \ldots, \overleftarrow{N}_{1}, 2 m\right] .
\end{aligned}
$$

After the zeros are removed, this is 


$$
\begin{aligned}
& {\left[\frac{(6 l+7)^{k}+3 l-1}{3}+2 ;\right.} 1,2 \frac{(6 l+7)^{k-1}-1}{3}, \vec{N}_{1}, \vec{N}_{2}, \ldots, \vec{N}_{k-2} \\
& \frac{(6 l+7)^{k-1}-1}{3}, 2, \frac{(6 l+7)^{k-2}-1}{3}+2,2, \frac{(6 l+7)^{k-1}-1}{3} \\
&\left.\overleftarrow{N}_{k-2}, \overleftarrow{N}_{k-3}, \ldots, \overleftarrow{N}_{2}, \overleftarrow{N}_{1}, 2 \frac{(6 l+7)^{k-2}-1}{3}+4\right]
\end{aligned}
$$

Since $r=6 l+7$ is odd, $d$ is not equivalent to $1(\bmod 4)$; so when $d$ is square free, we have the fundamental unit of $\mathbb{Q}(\sqrt{d})$ :

$$
\frac{\beta^{2 k}}{2 \alpha^{2}}
$$

where $\alpha=-\frac{(6 l+7)^{k}+3 l-1}{6}+\frac{1}{2} \sqrt{d}$ and $\beta=3+\frac{(6 l+7)^{k}+3 l-1}{6}+\frac{1}{2} \sqrt{d}$.

If we begin with

$$
N=\left\{a_{1} ; a_{2}, \ldots, a_{n}\right\}=\left(\begin{array}{cc}
u & v \\
2 v & w
\end{array}\right),
$$

then all the powers $N^{n}$ will have the same matrix form. We can use the Chebychev polynomials to express these powers explicitly.

First we recall how Chebychev polynomials can be used to deal with quadratic units. (See page 355 in $[\mathbf{L N}]$.) We begin with the identity

$$
x^{k}+y^{k}=\sum_{j=0}^{\left\lfloor\frac{k}{2}\right\rfloor} \frac{k}{k-j}\left(\begin{array}{c}
k \\
k-j
\end{array}\right)(-x y)^{j}(x+y)^{k-2 j} .
$$

If we have a quadratic unit $\beta$ with norm $\epsilon$,

$$
\beta^{k}+\bar{\beta}^{k}=\sum_{j=0}^{\left\lfloor\frac{k}{2}\right\rfloor} \frac{k}{k-j}\left(\begin{array}{c}
k \\
k-j
\end{array}\right)(-\epsilon)^{j}(\beta+\bar{\beta})^{k-2 j} .
$$

Therefore if $\beta=s+t \sqrt{d}$ has norm 1

$$
\beta^{k}+\bar{\beta}^{k}=\sum_{j=0}^{\left\lfloor\frac{k}{2}\right\rfloor} \frac{k}{k-j}\left(\begin{array}{c}
k \\
k-j
\end{array}\right)(-1)^{j}(2 s)^{k-2 j}=2 T_{k}(s)
$$

where $T_{k}(s)$ is the Chebychev polynomial of the first kind of degree $k$.

If

$$
N=\left\{a_{1} ; a_{2}, \ldots, a_{n}\right\}=\left(\begin{array}{cc}
u & v \\
2 v & w
\end{array}\right)
$$

has determinant one, $N^{n}$ can be explicitly given in terms of the rational part of the eigenvalues of $N$. Let

$$
\beta=\frac{1}{2}\left(u+w+\sqrt{(u+w)^{2}-4}\right)
$$


and

$$
\bar{\beta}=\frac{1}{2}\left(u+w-\sqrt{(u+w)^{2}-4}\right)
$$

be the eigenvalues, and note that $\beta \bar{\beta}=1$. Then

$$
N^{n}=\left(\begin{array}{cc}
U & V \\
2 V & W
\end{array}\right)
$$

where

$$
\begin{aligned}
U & =\frac{\left(\beta^{n-2}+\bar{\beta}^{n-2}\right)-\left(\beta^{n}+\bar{\beta}^{n}\right)-u\left(\beta^{n-1}+\bar{\beta}^{n-1}\right)+u\left(\beta^{n+1}+\bar{\beta}^{n+1}\right)}{(u+v)^{2}-4} \\
V & =\frac{v\left(\beta^{n+1}+\bar{\beta}^{n+1}\right)-v\left(\beta^{n-1}+\bar{\beta}^{n-1}\right)}{(u+w)^{2}-4} \\
W & =\frac{\left(\beta^{n+2}+\bar{\beta}^{n+2}\right)-\left(\beta^{n}+\bar{\beta}^{n}\right)-u\left(\beta^{n+1}+\bar{\beta}^{n+1}\right)+u\left(\beta^{n-1}+\bar{\beta}^{n-1}\right)}{(u+v)^{2}-4} .
\end{aligned}
$$

So

$$
\begin{aligned}
U & =\frac{2 T_{n-2}\left(\frac{u+w}{2}\right)-2 T_{n}\left(\frac{u+w}{2}\right)-2 u T_{n-1}\left(\frac{u+w}{2}\right)+2 u T_{n+1}\left(\frac{u+w}{2}\right)}{(u+w)^{2}-4} \\
V & =\frac{2 v\left(T_{n+1}\left(\frac{u+w}{2}\right)-T_{n-1}\left(\frac{u+w}{2}\right)\right)}{(u+w)^{2}-4} \\
W & =\frac{2 T_{n+2}\left(\frac{u+w}{2}\right)-2 T_{n}\left(\frac{u+w}{2}\right)-2 u T_{n+1}\left(\frac{u+w}{2}\right)+2 u T_{n-1}\left(\frac{u+w}{2}\right)}{(u+w)^{2}-4} .
\end{aligned}
$$

With these two expressions we can use Proposition 2. If we choose $l=0, r$ will simplify just a bit:

$$
\begin{aligned}
r & =W^{2}-2 V^{2} \\
& =\frac{4 v^{2}+T_{2 n+2}\left(\frac{u+w}{2}\right)-2 u T_{2 n}\left(\frac{u+w}{2}\right)+\left(u^{2}-2 v^{2}\right) T_{2 n+1}\left(\frac{u+w}{2}\right)}{(u+v)^{2}-4} .
\end{aligned}
$$

After this we use the values set in Proposition 2:

$$
\begin{aligned}
A_{i} & =\frac{r^{i}-1}{W} \\
B_{i} & =2 V A_{i} \\
m & =V A_{k}
\end{aligned}
$$

and

$$
d=d(u, v, w, \delta ; l, k)=m^{2}+2 r^{k} .
$$


Then the continued fraction expansion of $\sqrt{d}$ is

$$
\begin{aligned}
& {\left[m, \quad v A_{0}, \vec{N}, \vec{N}, \vec{N}, \ldots, \vec{N}, B_{k-1},\right.} \\
& v A_{1}, \vec{N}, \vec{N}, \vec{N}, \ldots, \vec{N}, B_{k-2} \\
& v A_{k-1}, \vec{N}, \vec{N}, \vec{N}, \ldots, \vec{N},, B_{0}, \\
& m, \quad B_{0}, \overleftarrow{N}, \overleftarrow{N}, \overleftarrow{N}, \ldots, \overleftarrow{N}, v A_{k-1} \\
& \left.B_{0}, \overleftarrow{N}, \overleftarrow{N}, \overleftarrow{N}, \ldots, \overleftarrow{N}, v A_{0}, 2 m\right]
\end{aligned}
$$

where each $N$ appears $n$ times.

In particular, if we begin with

$$
N=\left(\begin{array}{ll}
1 & 1 \\
2 & 3
\end{array}\right),
$$

then

$$
\begin{aligned}
U & =\frac{T_{n-2}(2)-T_{n}(2)-T_{n-1}(2)+T_{n+1}(2)}{6} \\
V & =\frac{T_{n+1}(2)-T_{n-1}(2)}{6} \\
W & =\frac{T_{n+2}(2)-T_{n}(2)-T_{n+1}(2)+T_{n-1}(2)}{6} \\
r & =\frac{4+T_{n+2}(2)-2 T_{2 n}(2)-2 T_{2 n+1}(2)}{12} .
\end{aligned}
$$

After this we use the values set in Proposition 2:

$$
\begin{aligned}
A_{i} & =\frac{r^{i}-1}{W} \\
B_{i} & =2 V A_{i} \\
m & =V A_{k}
\end{aligned}
$$

and

$$
d=d(u, v, w, \delta ; l, k)=m^{2}+2 r^{k} .
$$

This leads to

$$
\begin{aligned}
& \sqrt{d}=\left[A_{k} ; \quad A_{0}, \quad 1,2,1,2, \ldots \ldots \ldots, 1,2, B_{k-1},\right. \\
& A_{1}, \quad 1,2,1,2, \ldots \ldots \ldots, 1,2, B_{k-2} \text {, } \\
& A_{2}, \quad 1,2,1,2, \ldots \ldots \ldots, 1,2, B_{k-3},
\end{aligned}
$$




$$
\begin{aligned}
& A_{k-2}, 1,2,1,2, \ldots \ldots \ldots, 1,2, B_{1}, \\
& A_{k-1}, 1,2,1,2, \ldots \ldots \ldots, 1,2, B_{1}, \\
& A_{k}, \\
& B_{0} \quad 2,1,2, \ldots \ldots \ldots, 2,1, A_{k-1}, \\
& B_{1} \quad 2,1,2, \ldots \ldots \ldots, 2,1, A_{k-2}, \\
& B_{2} \quad 2,1,2, \ldots \ldots \ldots, 2,1, A_{k-3}, \\
& \ldots \ldots \ldots \ldots \ldots \ldots \ldots \ldots, 2,1, A_{1}, \\
& B_{k-2}, 2,1,2, \ldots \ldots \ldots, 1, A_{0}, \\
& B_{k-1}, 2,1,2, \ldots \ldots \ldots \ldots \ldots \\
& \left.2 A_{k}\right]
\end{aligned}
$$

where in each line the $(1,2)$ pair appears $n$ times. After the zeros are removed the repeating part has length $2 k(2 n+2)-2$.

\section{Section 7.}

The next technique for finding sequences to start the process involves factoring $2 v^{1} \pm 1$. To begin, factor $2 v^{2}+\epsilon=w w_{1}$. Write

$$
\frac{w}{v}=\left[a_{1} ; a_{2}, \ldots, a_{n}\right] .
$$

We can arrange it so that $(-1)^{n}=\epsilon$. Now

$$
\left(\begin{array}{cc}
0 & 1 \\
1 & a_{1}
\end{array}\right)\left(\begin{array}{cc}
0 & 1 \\
1 & a_{2}
\end{array}\right) \cdots\left(\begin{array}{cc}
0 & 1 \\
1 & a_{n}
\end{array}\right)=\left(\begin{array}{cc}
u & v \\
x & w
\end{array}\right) .
$$

Since $u w-x v=\epsilon$, and $w w_{1}-2 v^{2}=\epsilon$. So $x \equiv 2 v(\bmod w)$. This leads us to Proposition 2.

In practice, this method of producing sequences $a_{1}, a_{2}, \ldots, a_{n}$ can be used to produce very explicit, yet complicated, families. For example, let us start with the prime 3 ; if $v \equiv 2(\bmod 3)$, then $2 v^{2}+1 \equiv 0 \bmod 3 .(\epsilon=1$.) Let $v=2+3 n$. Choose

$$
w=\frac{2 v^{2}+1}{3}=6 n^{2}+8 n+3 .
$$

Then

$$
\begin{aligned}
\frac{w}{v}=\frac{6 n^{2}+8 n+3}{3 n+2} & =2 n+1+\frac{6 n^{2}+8 n+3-6 n^{2}-7 n-2}{3 n+2} \\
& =2 n+1+\frac{n+1}{3 n+2} \\
\frac{3 n+2}{n+1} & =2+\frac{n}{n+1}
\end{aligned}
$$




$$
\begin{gathered}
\frac{n+1}{n}=1+\frac{1}{n} \\
\frac{n}{1}=n .
\end{gathered}
$$

Thus

$$
\frac{6 n^{2}+8 n+3}{3 n+2}=[2 n+1,2,1, n] .
$$

The associated matrix is

$$
\left(\begin{array}{cc}
u & v \\
2 v & w
\end{array}\right)=\left(\begin{array}{cc}
3 n-1 & 3 n+2 \\
6 n+4 & 6 n^{2}+8 n+3
\end{array}\right) .
$$

Now we can set things up according to Proposition 2,

$$
r=w^{2}-2 v^{2}+2 l w v .
$$

Since

$$
\frac{w}{v}=\frac{6 n^{2}+8 n+3}{3 n+2}=2 n+1+\frac{n+1}{3 n+2}>2,
$$

we can even take $l=0$. Returning to Proposition 2,

$$
\begin{gathered}
r=36 n^{4}+(96+36 l) n^{3}+(82+72 l) n^{2}+(24+50 l) n+(1+12 l) \\
A_{i}=\frac{r^{i}-1}{w} \\
B_{i}=2(3 n+2) A_{i} \\
m=(3 n+2) A_{k}+(3 n+2) l
\end{gathered}
$$

and

$$
d=m^{2}+2 r^{k} .
$$

Then

$$
\begin{aligned}
& \sqrt{d}=\left[m+2 n+1 ; \quad 2,1, n, B_{k-1},\right. \\
& A_{1}, \quad(2 n+1), 2,1, n, B_{k-2}, \\
& A_{2}, \quad(2 n+1), 2,1, n, B_{k-3}, \\
& A_{k-2},(2 n+1), 2,1, n, B_{1} \text {, } \\
& A_{k-1},(2 n+1), 2,1 \text {, } \\
& 2 n+m+1 \text {, } \\
& 2,(2 n+1), A_{k-1}, \\
& B_{1}, \quad n, 1,2,(2 n+1), A_{k-2} \text {, } \\
& B_{2}, \quad n, 1,2,(2 n+1), A_{k-3} \text {, } \\
& B_{k-2}, n, 1,2,(2 n+1), A_{1} \text {, }
\end{aligned}
$$




$$
\begin{aligned}
& B_{k-1}, n, 1,2, \\
& 4 n+2+m] .
\end{aligned}
$$

\section{Section 8 .}

The final method of constructing continued fractions allows us to produce very intricate expansions by embedding the ones constructed above inside recurring patterns within the repeating quotients of larger fractions. As we have seen, the key to such embeddings is finding finite continued fractions $\left[a_{1}, a_{2}, \ldots \ldots, a_{n}\right]$ that lead to fractions of the form

$$
\left(\begin{array}{cc}
u & v \\
2 v-\delta w & w
\end{array}\right)
$$

with $\delta=0$ or 1 . As it turns out, the first half of all the repeating patterns above will do this.

Let us return to the calculation (and the notation) of Section 1. We started with a family of matrices

$$
N_{i}=\left(\begin{array}{cc}
p & 2 b r^{i} \\
b r^{k-1-i} & q
\end{array}\right) .
$$

Without going into the details of the calculation, a return to original identity shows that

$$
\left(N_{k-1} \ldots N_{1} N_{0}\right)^{T}
$$

has the form

$$
\left(\begin{array}{cc}
u & v \\
2 v & w
\end{array}\right) .
$$

Since the $N_{t}$ are products of matrices from continued fractions, we can embed the associated sequence of partial quotients inside a complementary pair $A_{i}, B_{i}$ to recur any number of times within the repeating pattern.

Returning to our first example:

$$
\begin{aligned}
& \sqrt{\left(b(2 b l+1)^{k}+l\right)^{2}+2(2 b l+1)^{k}} \\
&=\left[b(2 b l+1)^{k}+l ;\right. \\
& \quad b, 2 b(2 b l+1)^{k-1}, b(2 b l+1)^{k-2}, b(2 b l+1)^{2}, \ldots, \\
& \quad b(2 b l+1)^{k-2}, 2 b(2 b l+1)^{1}, b(2 b l+1)^{k-1}, 2 b, \\
& \quad b(2 b l+1)^{k}+l, \\
& \quad 2 b, b(2 b l+1)^{k-1}, 2 b(2 b l+1), b(2 b l+1)^{k-2}, 2 b(2 b l+1)^{2}, \ldots, \\
&\left.2 b(2 b l+1)^{k-2}, b(2 b l+1)^{1}, 2 b(2 b l+1)^{k-1}, b, 2 b(2 b l+1)^{k}+2 l\right] .
\end{aligned}
$$


If we take $b=1$ and $l=5,($ and $k=n)$, we get

$$
\begin{aligned}
& \sqrt{11^{2 n}+12 \cdot 11^{n}+25} \\
= & {\left[11^{n}+5 ;\right.} \\
& 1,2 \cdot 11^{n-1}, 11,2 \cdot 11^{n-2}, 11^{2}, 2 \cdot 11^{n-3}, \ldots, 11^{n-2}, 2 \cdot 11,11^{n-1}, 2, \\
& 11^{n}+5, \\
& 2,11^{n-1}, 2 \cdot 11,11^{n-2}, 2 \cdot 11^{2}, \ldots, 2 \cdot 11^{n-2}, 11,2 \cdot 11^{n-1}, 1, \\
& \left.2 \cdot 11^{n}+10\right] .
\end{aligned}
$$

And in the notation above

$$
\beta=6+11^{n}+\sqrt{11^{2 n}+12 \cdot 11^{n}+25} .
$$

Then according to the calculation above

$$
\begin{aligned}
& \left\{1,2 \cdot 11^{n-1}, 11,2 \cdot 11^{n-2}, 11^{2}, 2 \cdot 11^{n-3}, \ldots 11^{n-2}, 2 \cdot 11,11^{n-1}, 2\right\} \\
& =\left(\begin{array}{cc}
s-m t & 2 t \\
t & (11)^{-n}(s+m t)
\end{array}\right)=U_{n}
\end{aligned}
$$

where $\beta^{n}=s+t \sqrt{11^{2 n}+12 \cdot 11^{n}+25}$. If we now reset our notation and use Proposition 2 and set $l=0$, we define

$$
\begin{aligned}
\epsilon & =1 \\
r & =w^{2}-2 v^{2} \\
& =\left(s^{2}+2\left(11^{n}+5\right) s t+\left(25+10 \cdot 11^{n}-11^{2 n}\right) t^{2}\right) / 11^{2 n} \\
A_{i} & =\frac{r^{i}-1}{w} \\
B_{i} & =2 v A_{i}+\delta \\
m_{2} & =v A_{k}+\epsilon l
\end{aligned}
$$

and

$$
d=m_{2}^{2}+2 r^{k}=v^{2} A_{k}^{2}+2(\epsilon l v+w) A_{k}+2 .
$$

Then

$$
\begin{aligned}
& \sqrt{d}=\left[a_{k} ; a_{0}, \overleftarrow{U}_{n}, B_{k-1}, A_{1}, \overleftarrow{U}_{n}, B_{k-2}, \ldots, A_{k-1}, \overleftarrow{U}_{n}, B_{0}, A_{k}\right. \\
& \left.B_{0}, \vec{U}_{n}, A_{k-1}, B_{0}, \vec{U}_{n}, A_{k-1}, \ldots, B_{0}, \vec{U}_{n}, A_{k-1}, 2 A_{k}\right]
\end{aligned}
$$

After removing the zeros, the repeating part of this expansion has length $2 k(2 n+2)-2$. 


\section{Section 9.}

We conclude with a few specific examples that can be constructed using our techniques. Let

$$
\begin{aligned}
r & =2473892093033277097181734801 \\
A_{t}^{\prime} & =863109604528081677152276000 \sum_{i=0}^{t-1} r^{i} \\
d & =A_{k}^{\prime 2}+2 r^{k} .
\end{aligned}
$$

Let $U=\{1,2,3,4,5,6,7,8,9,10\} ; V=\{5,18,4,14,3,10,2,6\}$. Then

$$
\begin{aligned}
\sqrt{d}= & {\left[A_{k}^{\prime} ; A_{0}^{\prime}, \vec{U}, \vec{V}, 2 A_{k-1}^{\prime}, A_{1}^{\prime}, \vec{U}, \vec{V}, 2 A_{k-2}^{\prime}, \ldots, A_{k-1}^{\prime}, \vec{U}, \vec{V},, 2 A_{0}^{\prime}\right.} \\
& \left.A_{k}^{\prime}, 2 A_{0}^{\prime}, \overleftarrow{V}, \overleftarrow{U}, A_{k-1}^{\prime}, 2 A_{1}^{\prime}, \overleftarrow{V}, \overleftarrow{U}, A_{k-2}^{\prime}, \ldots, 2 A_{k-1}^{\prime}, \overleftarrow{V}, \overleftarrow{U}, A_{0}^{\prime}, 2 A_{k}^{\prime}\right]
\end{aligned}
$$

Next consider

$$
U=\{1,10,1,4,1,\}=\left(\begin{array}{cc}
54 & 65 \\
2 \cdot 65-71 & 71
\end{array}\right) .
$$

This is an example of a matrix with, in the notation we have been using, $\delta=1$. Using Proposition 2,

$$
\begin{aligned}
\epsilon & =-1 \\
r & =9230 l+3409 \\
A_{i} & =(130 l+48) \sum_{j=0}^{i-1}(9230 l+3409)^{j} \\
B_{i} & =130 A_{i}+1 \\
m & =130 A_{k}-l
\end{aligned}
$$

and

$$
d=(71)^{-2}\left(16900 r^{2 k}-(9230 l+23718) r^{k}+\left(5041 l^{2}+9230 l+16900\right)\right) .
$$

Then

$$
\begin{aligned}
\sqrt{d}=\left[m, v A_{0}, \vec{U}, B_{k-1}, v A_{1}, \vec{U}, B_{k-2}, \ldots, v A_{k-1}, \vec{U}, B_{0}\right. \\
\\
\left.m, B_{0}, \overleftarrow{U}, v A_{k-1}, \ldots, B_{k-1}, \overleftarrow{U}, v A_{0}, 2 m\right] .
\end{aligned}
$$

Next consider our very first family:

$$
\begin{aligned}
& \sqrt{\left(b(2 b n+1)^{k}+n\right)^{2}+2(2 b n+1)^{k}} \\
& =\left[b,(2 b n+1)^{k}+n ;\right.
\end{aligned}
$$




$$
\begin{aligned}
& b, 2 b(2 b n+1)^{k-1}, b(2 b n+1), 2 b(2 b n+1)^{k-2}, b(2 b n+1)^{2}, \ldots, \\
& b(2 b n+1)^{k-1}, b(2 b n+1)^{1}, 2 b(2 b n+1)^{k-1}, 2 b, \\
& b(2 b n+1)^{k}+n, \\
& 2 b, b(2 b n+1)^{k-1}, 2 b(2 b n+1), b(2 b n+1)^{k-2}, 2 b(2 b n+1)^{2}, \ldots, \\
& \left.2 b(2 b n+1)^{k-2}, b(2 b n+1)^{1}, 2 b(2 b n+1)^{k-1}, b, 2 b(2 b n+1)^{k}+2 n\right] .
\end{aligned}
$$

If we take $n=b$, we get

$$
\begin{aligned}
& \sqrt{b^{2}\left(\left(2 b^{2}+1\right)^{k}+1\right)^{2}+2\left(2 b^{2}+1\right)^{k}} \\
&=\sqrt{b^{2}\left(2 b^{2}+1\right)^{2 k}+2\left(b^{2}+1\right)\left(2 b^{2}+1\right)^{k}+1} \\
&=\left[b\left(2 b^{2}+1\right)^{k}+b ;\right. \\
& \quad b, 2 b\left(2 b^{2}+1\right)^{k-1}, b\left(2 b^{2}+1\right), 2 b\left(2 b^{2}+1\right)^{k-2}, b\left(2 b^{2}+1\right)^{2}, \ldots, \\
& \quad b\left(2 b^{2}+1\right)^{k-2}, 2 b\left(2 b^{2}+1\right)^{1}, b\left(2 b^{2}+1\right)^{k-1}, 2 b, \\
& \quad b\left(2 b^{2}+1\right)^{k}+b, \\
& 2 b, b\left(2 b^{2}+1\right)^{k-1}, 2 b\left(2 b^{2}+1\right), b\left(2 b^{2}+1\right)^{k-2}, 2 b\left(2 b^{2}+1\right)^{2}, \ldots, \\
&\left.2 b\left(2 b^{2}+1\right)^{k-2}, b\left(2 b^{2}+1\right)^{1}, 2 b\left(2 b^{2}+1\right)^{k-1}, b, 2 b\left(2 b^{2}+1\right)^{k}+2 b\right] .
\end{aligned}
$$

We can multiply this by $b$ to get a new expansion:

$$
\begin{aligned}
\sqrt{b^{4}\left(2 b^{2}+1\right)^{2 k}+2 b^{2}\left(b^{2}+1\right)\left(2 b^{2}+1\right)^{k}+b^{2}} \\
=\left[b^{2}\left(2 b^{2}+1\right)^{k}+b^{2} ;\right. \\
\quad 1,2 b^{2}\left(2 b^{2}+1\right)^{k-1},\left(2 b^{2}+1\right), 2 b^{2}\left(2 b^{2}+1\right)^{k-2},\left(2 b^{2}+1\right)^{2}, \ldots, \\
\quad\left(2 b^{2}+1\right)^{k-2}, 2 b^{2}\left(2 b^{2}+1\right)^{1},\left(2 b^{2}+1\right)^{k-1}, 2 b^{2}, \\
\quad\left(2 b^{2}+1\right)^{k}+1, \\
2 b^{2},\left(2 b^{2}+1\right)^{k-1}, 2 b^{2}\left(2 b^{2}+1\right),\left(2 b^{2}+1\right)^{k-2}, 2 b^{2}\left(2 b^{2}+1\right)^{2}, \ldots, \\
\left.2 b^{2}\left(2 b^{2}+1\right)^{k-2},\left(2 b^{2}+1\right)^{1}, 2 b^{2}\left(2 b^{2}+1\right)^{k-1}, 1,2 b^{2}\left(2 b^{2}+1\right)^{k}+2 b^{2}\right] .
\end{aligned}
$$

\section{References}

[A] T. Azuhatu, On the fundamental units and class numbers of real quadratic fields, II, Tokyo Journal of Math., 10 (1987), 259-270.

[LR] C. Levesque and G. Rind, A few classes of periodic continued fractions, Utilitas Math, 30 (1986), 79-107. 
[LN] R. Lidl and Niederreiter, Finite Fields, Addison-Wesley Publishing Co. Reading, Massachusetts, 1983.

[vdP] A.J. van der Poorten, Explicit formulas for units in certain quadratic number fields, Algorithmic Number Theory (Ithaca, NY, 1994), Lecture Notes in Computer Science, 877, Springer, Berlin, (1994), 194-208.

[W] H.C. Williams, A note on the periodic length of the continued fraction length of certain $\sqrt{D}$, Utilitas Math., 28 (1985), 201-209.

Received October 9, 1998 and revised November 15, 1999.

UNIVERSITY OF ARIZONA

TuCson Arizona 85721

E-mail address: madden@math.arizona.edu 